The paper is presented at the conference "Complex analysis and its applications" (COMAN 2018), Gelendzhik - Krasnodar, Russia, June 2-9, 2018.

UDC 517.518

N. A. Kudryavtseva, S. K. Vodopyanov

\title{
ON THE CONVERGENCE OF MAPPINGS WITH k-FINITE DISTORTION.
}

\begin{abstract}
We prove that a locally uniform limit of a sequence of homeomorphisms with finite $k$-distortion is also a mapping with finite $k$-distortion. We obtain also an estimation for the distortion coefficient of the limit mapping.
\end{abstract}

Key words: mapping with $k$-finite distortion, distortion coeffcient, passing to the limit, differential form.

\section{Mathematical Subject Classification: 30C65}

1. Introduction. It is well known that the limit of a uniformly converging sequence of analytic functions is an analytic function. Reshetnyak generalized this result to mappings with bounded distortion: the limit of a locally uniformly converging sequence of mappings with bounded distortion is a mapping with bounded distortion.

Definition 1. [8] A mapping $f: \Omega \rightarrow \mathbb{R}^{n}$ is called mapping with bounded distortion if $f$ is continuous, $f \in W_{n \text {, loc }}^{1}(\Omega)$, the Jacobian $J(x, f)$ does not change the sign in the domain $\Omega$ and

$$
|D f(x)|^{n} \leqslant K|J(x, f)| \quad \text { for almost all } x \in \Omega .
$$

The smallest constant in this inequality is called the distortion coefficient of the mapping $f$ and is denoted by the symbol $K(f)$. It is clear that

$$
K(f)=\sup \left\{\frac{|D f(x)|^{n}}{|J(x, f)|}: \quad x \in \Omega, \quad J(x, f) \neq 0\right\} .
$$

Reshetnyak used the weak convergence of Jacobians to prove the following theorem on the limit of a sequence of mappings with bounded distortion.

(C) Petrozavodsk State University, 2018

\section{(cc) EY-NC}


Theorem 1. [8] Let $f_{m}: \Omega \rightarrow \mathbb{R}^{n}, m=1,2, \ldots$, be an arbitrary sequence of mappings with bounded distortion, locally converging in $L_{n}(\Omega)$ to a mapping $f_{0}: \Omega \rightarrow \mathbb{R}^{n}$. Assume that the sequence of distortion coefficients $K\left(f_{m}\right), m=1,2, \ldots$, is bounded. Then the limit mapping $f_{0}$ is a mapping with bounded distortion and the following inequality holds:

$$
K\left(f_{0}\right) \leqslant \varliminf_{k \rightarrow \infty} K\left(f_{m}\right) .
$$

We briefly outline the proof in the case of non-negative Jacobians. For a test function $\varphi \in C_{0}^{\infty}(\Omega)$ we have

$$
\begin{aligned}
& \int_{\Omega}\left|D f_{0}(x)\right|^{n} \varphi(x) d x \leqslant \underline{\lim }_{m \rightarrow \infty} \int_{\Omega}\left|D f_{m}(x)\right|^{n} \varphi(x) d x \leqslant \\
& \leqslant K \varliminf_{m \rightarrow \infty} \int_{\Omega} J\left(x, f_{m}\right) \varphi(x) d x=K \int_{\Omega} J\left(x, f_{0}\right) \varphi(x) d x .
\end{aligned}
$$

To justify the limit in the last equality, we apply the weak convergence of Jacobians. Consequently, for the limit mapping, the point-wise inequality $\left|D f_{0}(x)\right|^{n} \leqslant K J\left(x, f_{0}\right)$ holds a .e. in $\Omega$.

More recently, research has begun on mappings with finite distortion. They are a natural generalization of mappings with bounded distortion.

Definition 2. [7] Let a mapping $f: \Omega \rightarrow \mathbb{R}^{n}$ belong to the Sobolev class $W_{n, \mathrm{loc}}^{1}(\Omega)$ and $J(x, f) \geqslant 0$. We define the pointwise distortion coefficient $K(x, f)$ of the mapping $f$ as a value

$$
K(x, f)= \begin{cases}\frac{|D f(x)|^{n}}{|J(x, f)|} & \text { if } J(x, f)>0, \\ 1 & \text { otherwise. }\end{cases}
$$

The mapping $f: \Omega \rightarrow \mathbb{R}^{n}$ is called mapping with finite distortion $(f \in$ $\in F D(\Omega))$ if

$|D f(x)|^{n} \leqslant K(x, f) J(x, f)$ where $1 \leqslant K(x, f)<\infty$ for almost all $x \in \Omega$.

Remark. In other words, the condition of finite distortion is that the partial derivatives of the mapping $f \in W_{n, \text { loc }}^{1}(\Omega)$ vanish a.e. on the set of zeros of the Jacobian $J(x, f)$. 
For the first time, the essential properties of mappings with finite distortion were investigated in the paper [15] in the study of homeomorphisms inducing a bounded composition operator. The name "mapping with finite distortion" was proposed much later in the paper [7].

In [5] F. Gehring and T. Iwaniec showed that the limit of a weakly converging sequence of mappings with finite distortion is also a mapping with finite distortion, and they obtained also an estimation for the distortion coefficient of the limit mapping.

Theorem 2. [5] Let $f_{m}: \Omega \rightarrow \mathbb{R}^{n}, m=1,2, \ldots$, be an arbitrary sequence of mappings with finite distortion converging weakly in $W_{n, \mathrm{loc}}^{1}(\Omega)$ to a mapping $f_{0}: \Omega \rightarrow \mathbb{R}^{n}$. Assume that

$$
K\left(x, f_{m}\right) \leqslant M(x)<\infty, \quad m=1,2, \ldots,
$$

where $\Omega \ni x \mapsto M(x) \in[1, \infty]$ is a measurable function. Then the limit mapping $f_{0}$ is a mapping with finite distortion and the inequality

$$
K\left(x, f_{0}\right) \leqslant M(x)
$$

holds.

More precisely, in this paper existence of a subsequence $f_{m_{k}}$ such that

$$
K\left(x, f_{0}\right) \leqslant b * \lim _{k \rightarrow \infty} K\left(x, f_{m_{k}}\right)
$$

was shown. Here the limit is understood in the sense of so-called biting convergence.

Definition 3. [2] Let $h$ and $h_{k}, k \in \mathbb{N}$, be Lebesgue measurable functions defined on a set $E \subset \mathbb{R}^{n}$. The sequence $h_{k}$ is said to converge in the biting sense on $E$ to $h$ if there exists an increasing sequence $E_{\nu}$ of measurable subsets of $E$,

$$
\bigcup_{\nu} E_{\nu}=E
$$

such that

$$
\lim _{k \rightarrow \infty} \int_{E_{\nu}} \varphi h_{k} d x=\int_{E_{\nu}} \varphi h d x
$$

for any function $\varphi \in L_{\infty}\left(E_{\nu}\right)$. 
In the paper [4] the limit of a sequence of homeomorphisms with finite distortion converging weakly in $W_{1}^{1}$ was shown to have, also, a finite distortion under the condition that the limit mapping is a homeomorphism.

Theorem 3. [4] Let $\Omega, \Omega^{\prime}$ be bounded domains in $\mathbb{R}^{n}, f_{j}: \Omega \rightarrow \Omega^{\prime}$, $j \in \mathbb{N}, f: \Omega \rightarrow \Omega^{\prime}$, be homeomorpisms belonging to $W_{1}^{1}(\Omega)$, and $f_{j} \rightarrow f$ weakly in $W_{1}^{1}(\Omega)$. Assume that

$$
\left|D f_{j}(x)\right|^{n} \leqslant K\left(x, f_{j}\right) J\left(x, f_{j}\right) \quad \text { for almost all } x \in \Omega,
$$

where $K\left(x, f_{j}\right): \Omega \rightarrow[1, \infty)$ are Borel functions for all $j$, and the sequence $K\left(x, f_{j}\right)$ converges in the biting sense to $K(x)$ as $j \rightarrow \infty$. Then the limit mapping $f$ is a mapping with finite distortion and the inequality $K(x, f) \leqslant K(x)$ holds for almost all $x \in \Omega$.

In the paper [1] mappings with bounded $(q, p)$-distortion $(n-1<$ $<q \leqslant p<\infty)$ were defined and investigated; these mappings coincide with the class of mappings with bounded distortion if $q=p=n$.

In the paper [17] a locally uniform limit of a sequence of mappings with bounded $(\theta, 1)$-weighted $(q, p)$-distortion was shown to be, also, a mapping with a bounded $(\theta, 1)$-weighted $(q, p)$-distortion and an estimation similar to (2) was established. The proofs of the theorems like those in the article [17] and in this work are based on the method developed in [11] for extending Reshetnyak's result to Carnot groups.

In this paper we extend the above-mentioned assertions to the class of mappings with $k$-finite distortion, which arise naturally in the problem of operating with differential forms of degree $k$ (see [12]).

2. Preliminaries. Let $U$ be a domain in $\mathbb{R}^{n}$. We consider the Banach space $\mathcal{L}_{p}\left(U, \Lambda^{k}\right)$ of differential forms $\omega$ of degree $k, k=1, \ldots, n$, with measurable coefficients, which have the following finite norm: $\|\omega\|_{p}=$ $=\left(\int_{U}|\omega|^{p} d x\right)^{1 / p}$.

A mapping $f: U \rightarrow \mathbb{R}^{n}$ is said to be approximate differentiable at a point $x \in U$ [3], if there exists a linear mapping $L: \mathbb{R}^{n} \rightarrow \mathbb{R}^{n}$ such that

$$
\lim _{r \rightarrow 0} \frac{|\{y \in B(x, r):|f(y)-f(x)-L(y-x)|>\varepsilon\}|}{r^{n}}=0
$$

for any $\varepsilon>0$. Here the symbol $|\cdot|$ denotes the Lebesgue measure. It is well known that the approximate differential is unique [3] if $x$ is a density point. In what follows, it is denoted by the symbol ap $D f(x)$. 
In our paper we consider the mappings belonging to some Sobolev class. Such mappings are unconditionally approximately differentiable.

Let $\omega=\sum \omega_{\beta} d y^{\beta}$ be any $k$-form, $k=1, \ldots, n$, in $W$ with continuous coefficients $\omega_{\beta}: W \rightarrow \mathbb{R}$, where the summation is over all $k$-dimensional ordered multi-indices $\beta=\left(\beta_{1}, \ldots, \beta_{k}\right), 1 \leqslant \beta_{1}<\ldots<\beta_{k} \leqslant n$, and $d y^{\beta}=d y_{\beta_{1}} \wedge d y_{\beta_{2}} \wedge \ldots \wedge d y_{\beta_{k}}$. Let a mapping $f=\left(f_{1}, \ldots, f_{n}\right): U \rightarrow W$ of Euclidean domains $U, W \subset \mathbb{R}^{n}$ be approximately differentiable almost everywhere in $U$. We write the pull-back of the $k$-form $\omega$ in the following way:

$$
f^{*} \omega(x)=\sum_{\beta} \omega_{\beta}(f(x)) d f_{\beta_{1}} \wedge d f_{\beta_{2}} \wedge \ldots \wedge d f_{\beta_{k}}=\sum_{\alpha} \sum_{\beta} \omega_{\beta}(f(x)) M_{\alpha}^{\beta}(x) d x^{\alpha} .
$$

In other words, it is a $k$-form with measurable coefficients, which are defined for almost all $x \in U$ (here $d f_{\beta_{k}}=\sum_{i=1}^{n} \frac{\partial f_{\beta_{k}}}{\partial x_{i}} d x_{i}$ and the partial derivatives are understood in the approximate sense, $M_{\alpha}^{\beta}(x)$ are $(k \times k)$ minors of the matrix ap $D f(x)=\left(\frac{\partial f_{j}}{\partial x_{i}}\right), i, j=1, \ldots, n$, with ordered lines and columns).

We recall that the approximate differential ap $D f(x): T_{x} U \rightarrow T_{f(x)} W$ is defined a.e. in $U$. It generates canonically the linear mapping $\Lambda_{k} f(x): \Lambda_{k} T_{x} U \rightarrow \Lambda_{k} T_{f(x)} W$ of the spaces of $k$-vectors, and the pullback operation $f^{*}$ of $k$-forms. We denote the norm of the last linear mapping by the symbol $\left|\Lambda^{k} f(x)\right|$.

The minimal analytic and geometric properties of the mapping $f$ were obtained in [12] for generating a bounded pullback operator

$$
f^{*}: \mathcal{L}_{p}\left(W, \Lambda^{k}\right) \rightarrow \mathcal{L}_{q}\left(U, \Lambda^{k}\right), \quad 1 \leqslant q \leqslant p \leqslant \infty,
$$

of differential forms of degree $k=1, \ldots, n$.

We say that an approximately differentiable mapping $f: U \rightarrow W$ has $k$-finite distortion, $1 \leqslant k \leqslant n$, (shortly $f \in \mathcal{C D}^{k}(U ; W)$ ) if rank ap $D f(x)<$ $<k$ almost everywhere on a set $Z$. (Hereinafter $Z=\{x \in U: \operatorname{det}$ ap $D f(x)=$ $=0\}$.) For $k=1(k=n-1)$ and $f \in W_{1, \text { loc }}^{1}(U)\left(f \in W_{n-1, \mathrm{loc}}^{1}(U)\right)$, this notion is well-known in literature: it is just the class of Sobolev mappings with finite distortion (codistortion), which is characterized by the property: ap $D f(x)=0(\operatorname{adj} D f(x)=0)$ almost everywhere on $Z$ (see [10], [14] for the second notion).

Besides of the property of $k$-finite distortion we consider mappings with a certain behavior of some characteristics of the distortion containing in 
itself the ratio $\frac{\left|\Lambda^{k} f(x)\right|^{q}}{|J(x, f)|}$, where $J(x, f)=\operatorname{det} \operatorname{ap} D f(x)$ [12]: operator (4) is bounded if and only if the mapping $f \in \mathcal{C D}^{k}(U ; W)$ and the distortion function $\mathbb{W} \ni y \mapsto H_{k, q}(y)=$

$$
= \begin{cases}\left(\sum_{x \in f^{-1}(y) \backslash(\Sigma \cup Z)} \frac{\left|\Lambda^{k} f(x)\right|^{q}}{|J(x, f)|}\right)^{\frac{1}{q}} & \text { if } \quad f^{-1}(y) \backslash(\Sigma \cup Z) \neq \emptyset, \\ 0 & \text { otherwise, }\end{cases}
$$

belongs to $L_{\varkappa}(W)$ where $\frac{1}{\varkappa}=\frac{1}{q}-\frac{1}{p}$ if $q<p$, and $\varkappa=\infty(\varkappa=q)$ if $q=p$ $(p=\infty)$. Moreover, the norm of the operator $f^{*}$ is comparable with the value $\left\|H_{k, q}(\cdot) \mid L_{\varkappa}(W)\right\|$ :

$$
\alpha_{q, p}\left\|H_{k, q}(\cdot)\left|L_{\varkappa}(\Omega)\|\leqslant\| f^{*}\|\leqslant\| H_{k, q}(\cdot)\right| L_{\varkappa}(\Omega)\right\|
$$

where $\alpha_{q, p}$ is some constant.

Hereinafter $\Sigma \subset U$ is a set of measure zero outside of which the mapping $f$ has the Luzin property $\mathcal{N}$.

For homeomorphic mappings one can use a simpler characteristic.

Corollary 1. [12] Let $f: U \rightarrow W$ be an approximate differentiable homeomorphism. The operator $f^{*}: \mathcal{L}_{p}\left(W, \Lambda^{k}\right) \rightarrow \mathcal{L}_{q}\left(U \Lambda^{k}\right), 1 \leqslant q \leqslant p \leqslant \infty$, $k=1, \ldots, n$, is bounded if and only if the following conditions are satisfied:

1) $f: U \rightarrow W$ has the $k$-finite distortion;

2) the function $K_{k, p}(x, f)=\left\{\begin{array}{ll}\frac{\left|\Lambda^{k} f(x)\right|}{|J(x, f)|^{1 / p}} & \text { if } J(x, f) \neq 0, \\ 0 & \text { otherwise, }\end{array}\right.$ belongs to $L_{\varkappa}(U)$, where $\frac{1}{\varkappa}=\frac{1}{q}-\frac{1}{p}$ if $q<p$, and $\varkappa=\infty(\varkappa=q)$ if $q=p$ $(p=\infty)$.

In this case, the norm of the operator $f^{*}$ is comparable with

$$
\left\|K_{k, p}(\cdot, f)\left|L_{\varkappa}(U)\left\|: \alpha_{q, p}\right\| K_{k, p}(\cdot, f)\right| L_{\varkappa}(\Omega)\right\| \leqslant\left\|f^{*}\right\| \leqslant\left\|K_{k, p}(\cdot, f) \mid L_{\varkappa}(\Omega)\right\|,
$$

where $\alpha_{q, p}$ is some constant.

\section{Main results.}

Definition 4. [12] An approximately differentiable homeomorphism $f$ : $U \rightarrow W$ belongs to the class $\mathcal{C D}_{q, p}^{k}(U ; W)$ if the following conditions hold

1) $f \in \mathcal{C D}^{k}(U ; W)$; 
2) $K_{k, p}(\cdot, f) \in L_{\varkappa}(U)$ where $\frac{1}{\varkappa}=\frac{1}{q}-\frac{1}{p}, 1 \leqslant q \leqslant p<\infty$.

Theorem 4. Let $f_{m} \in \mathcal{C D}_{q, p}^{k}(U ; W), m \in \mathbb{N}$, be a sequence of homeomorphisms of the Sobolev class $W_{l, \mathrm{loc}}^{1}(U)$ with $k<l, q \leqslant l / k, 1<q \leqslant p<$ $<\infty$. Suppose that the sequence $f_{m}$ is locally bounded in $W_{l}^{1}(U)$, and locally uniformly converges to a homeomorphism $f: U \rightarrow W$ as $m \rightarrow \infty$. Assume also that there exists a sequence of functions $U \ni x \mapsto M_{m}(x)$, belonging to $L_{\varkappa}(U)$, that is bounded in $L_{\varkappa}(U), \frac{1}{\varkappa}=\frac{1}{q}-\frac{1}{p}$, for which the inequality

$$
K_{k, p}\left(x, f_{m}\right) \leqslant M_{m}(x) \text { for almost all } x \in U
$$

is true.

Then there exists a function $U \ni x \mapsto M(x)$ of $L_{\varkappa}(U)$ such that some subsequence

(i) in the case $1<q<p<\infty$ : of functions $\left\{M_{m}(x)^{\varkappa}\right\}_{m \in \mathbb{N}}$ converges in the biting sense to $M(x)^{\varkappa}$;

(ii) in the case $1<q=p<\infty$ : of numbers $\left\{\left\|M_{m} \mid L_{\infty}(U)\right\|\right\}_{m \in \mathbb{N}}$ converges to $M=\varliminf_{m \rightarrow \infty}\left\|M_{m} \mid L_{\infty}(U)\right\|$;

the limit mapping $f$ belongs to $\mathcal{C D}_{q, p}^{k}(U ; W)$ and $K_{k, p}(\cdot, f) \in L_{\varkappa}(U)$, where $\frac{1}{\varkappa}=\frac{1}{q}-\frac{1}{p}$.

Moreover, the inequalities

$$
\left\{\begin{array}{l}
K_{k, p}(x, f) \leqslant M(x) \text { in the case } q<p, \\
K_{k, p}(x, f) \leqslant M \text { in the case } q=p
\end{array}\right.
$$

hold for almost all $x \in U$.

In the proof we use some arguments from the paper [17], where Theorem 4 is proved for $k=1$.

Proof. It follows from the conditions of the theorem that $f \in W_{l, \mathrm{loc}}^{1}(U)$. First, we show that the limit mapping $f$ belongs to $\mathcal{C D}^{k}(U ; W)$. For doing this, we show that the mapping $f$ induces a bounded operator $f^{*}: \mathcal{L}_{p}\left(W, \Lambda^{k}\right) \rightarrow \mathcal{L}_{q}\left(U, \Lambda^{k}\right), 1<q \leqslant p<\infty$. Since every mapping $f_{m} \in \mathcal{C D}_{q, p}^{k}(U ; W)$, it follows from Corollary 1 that the homeomorphism $f_{m}: U \rightarrow W$ induces the bounded operator $f_{m}^{*}: \mathcal{L}_{p}\left(W, \Lambda^{k}\right) \rightarrow \mathcal{L}_{q}\left(U, \Lambda^{k}\right)$, $1<q \leqslant p<\infty, m \in \mathbb{N}$. Moreover, the norms of the operators $f_{m}^{*}$ are totally bounded

$$
\left\|f_{m}^{*}\right\| \leqslant\left\|K_{k, p}\left(\cdot, f_{m}\right)\left|L_{\varkappa}(U)\|\leqslant\| M_{m}(\cdot)\right| L_{\varkappa}(U)\right\| \leqslant \tilde{M}<\infty .
$$


Take a $k$-form $\omega \in \mathcal{L}_{p}\left(W, \Lambda^{k}\right) \cap \mathcal{C}\left(W, \Lambda^{k}\right)$ and set $\sigma_{m}=f_{m}^{*}(\omega)$. Since $\left\|f_{m}^{*}\right\| \leqslant \tilde{M}$, the sequence of forms $\sigma_{m}$ is bounded in $\mathcal{L}_{q}\left(U, \Lambda^{k}\right)$. Therefore, we can extract a weakly converging subsequence. We assume that the sequence $\sigma_{m}$ converges weakly in $\mathcal{L}_{q}\left(U, \Lambda^{k}\right)$ to a form $\sigma_{0}$. The weak convergence of forms means that coefficients of the forms $\sigma_{m}$ converge weakly in $L_{q}(U)$ to the corresponding coefficients of the form $\sigma_{0}$. Since the sequence $\sigma_{m}$ converges weakly in $\mathcal{L}_{q}\left(U, \Lambda^{k}\right)$ to $\sigma_{0}$ as $m \rightarrow \infty$, we have

$$
\begin{aligned}
\left\|\sigma_{0} \mid L_{q}(U)\right\| \leqslant \varliminf_{m \rightarrow \infty} & \left\|\sigma_{m}\left|L_{q}(U)\left\|=\varliminf_{m \rightarrow \infty}\right\| f_{m}^{*} \omega\right| L_{q}(U)\right\| \leqslant \\
& \leqslant \varliminf_{k \rightarrow \infty}\left\|f_{m}^{*}\right\| \cdot\left\|\omega\left|L_{p}(U)\|\leqslant \tilde{M} \cdot\| \omega\right| L_{p}(U)\right\| .
\end{aligned}
$$

The following lemma is proved in the book $[8$, Chapter $2, \S 4]$.

Lemma 1. [8] Suppose $U$ is an open subset in $\mathbb{R}^{n}$, and suppose that $\varphi_{m}=\left(\varphi_{m 1}, \varphi_{m 2}, \ldots, \varphi_{m k}\right), 1 \leqslant k \leqslant n, m=1,2, \ldots$, is a sequence of vector-functions of $W_{l, \mathrm{loc}}^{1}(U), k \leqslant l$, locally bounded in $W_{l}^{1}(U)$. Assume that, as $m \rightarrow \infty$, the functions $\varphi_{m}$ converge in $L_{1, \text { loc }}$ to a vector function $\varphi_{0}=\left(\varphi_{01}, \varphi_{02}, \ldots, \varphi_{0 k}\right)$, and set $\omega_{m}=d \varphi_{m 1} \wedge d \varphi_{m 2} \wedge \ldots \wedge d \varphi_{m k}$. Then the sequence of forms $\omega_{m}$ weakly converges in $L_{l / k, \text { loc }}(U)$ to a form $\omega_{0}{ }^{1}$.

Since the homeomorphisms $f_{m}$ locally uniformly converge to $f$ and the form $\omega$ has continuous coefficients, the functions $\omega_{\beta}\left(f_{m}(x)\right)$ converge locally uniformly to $\omega_{\beta}(f(x))$ as $m \rightarrow \infty$. Lemma 1 implies that the minors of the matrices $D f_{m}$ converge weakly in $L_{l / k, \text { loc }}(U)$ to minors of the matrix $D f$. Therefore, the forms $\sigma_{m}$ converge weakly in $L_{l / k \text {,loc }}(U)$ to $f^{*}(\omega)$.

It is not hard to see that both limits $\sigma_{0}$ and $f^{*}(\omega)$ coincide: $\sigma_{0}=$ $=f^{*}(\omega)$. In view of (6) the mapping $f$ induces a bounded operator $f^{*}$ : $\mathcal{L}_{p}\left(W, \Lambda^{k}\right) \rightarrow \mathcal{L}_{q}\left(U, \Lambda^{k}\right), 1<q \leqslant p<\infty$. By Corollary $1, f \in \mathcal{C D}^{k}(U ; W)$.

First, we consider the case $q<p$. The following lemma is valid.

Lemma 2. [2] Every sequence of mappings $h_{m}, m=1,2, \ldots$, that is bounded in $L_{1}(U)$, contains a subsequence, converging in the biting sense to some function $h \in L_{1}(U)$.

This lemma implies existence of a function $U \ni x \mapsto M(x)$ of $L_{\varkappa}(U)$ such that some subsequence of the function $h_{m}=M_{m}(x)^{\varkappa}$ converges

\footnotetext{
${ }^{1}$ It means that the sequence of forms $\omega_{m}$ converges weakly in $L_{l / k}(D)$ to a form $\omega_{0}$ on every subdomain $D \Subset U$.
} 
in the biting sense to $h=M(x)^{\varkappa}$. We assume that the given sequence $M_{m}(x)^{\varkappa}$ converges in the biting sense to the function $M(x)^{\varkappa}$ (the set of the Definition 3 is denoted by $E_{\nu}$ ).

Now we estimate the distortion coefficient of the limit mapping $f$. For this, we consider estimates on a set $E_{\nu}$. Let $Z_{m}$ be the set of zeros of the Jacobian of the mapping $f_{m}$. Since the rank of the matrix $D f_{m}$ on the set $Z_{m}$ is less then $k$, it follows that all $k$-th-order minors are equal to zero on the set $Z_{m}$.

Applying the Hölder inequality, and taking into account that $\frac{q}{\varkappa}+\frac{q}{p}=1$ on each intersection $E_{\nu} \cap B\left(x_{0}, r\right)$, where $x_{0} \in E_{\nu}, B\left(x_{0}, r\right) \Subset U$, in view of (5) we have

$$
\begin{gathered}
\int_{E_{\nu} \cap B\left(x_{0}, r\right)}\left|\Lambda^{k} f_{m}(x)\right|^{q} d x=\int_{\left(E_{\nu} \cap B\left(x_{0}, r\right)\right) \backslash Z_{m}} \frac{\left|\Lambda^{k} f_{m}(x)\right|^{q}}{\left|J\left(x, f_{m}\right)\right|^{\frac{q}{p}}}\left|J\left(x, f_{m}\right)\right|^{\frac{q}{p}} d x \leqslant \\
\leqslant\left(\int_{\left(E_{\nu} \cap B\left(x_{0}, r\right)\right) \backslash Z_{m}} \frac{\left|\Lambda^{k} f_{m}(x)\right|^{q \frac{\varkappa}{q}}}{\left|J\left(x, f_{m}\right)\right|^{\frac{q}{p} \frac{\varkappa}{q}}} d x\right)^{\frac{q}{\varkappa}}\left(\int_{E_{\nu} \cap B\left(x_{0}, r\right)}\left|J\left(x, f_{m}\right)\right|^{\frac{q}{p} \frac{p}{q}} d x\right)^{\frac{q}{p}}= \\
=\left(\int_{E_{\nu} \cap B\left(x_{0}, r\right)}\left(K_{k, p}\left(f_{m}\right)\right)^{\varkappa}(x) d x\right)^{\frac{q}{\varkappa}}\left(\int_{E_{\nu} \cap B\left(x_{0}, r\right)}\left|J\left(x, f_{m}\right)\right| d x\right)^{\frac{q}{p}} \leqslant \\
\leqslant\left(\int_{E_{\nu} \cap B\left(x_{0}, r\right)} M_{m}^{\varkappa}(x) d x\right)^{\frac{q}{\varkappa}}\left(\int_{B\left(x_{0}, r\right)}\left|J\left(x, f_{m}\right)\right| d x\right)^{\frac{q}{p}} .
\end{gathered}
$$

Elements of the matrix $\Lambda^{k}\left(f_{m}\right)(x)$ are the $k$-th-order minors of ap $D_{m} f(x)$. In view of Lemma 1 , they converge weakly in $L_{l / k, \text { loc }}(U)$ to elements of the matrix $\Lambda^{k}(f)(x)$. Since $q \leqslant l / k, \Lambda^{k}\left(f_{m}\right)(x)$ converges weakly in $L_{q \text {, loc }}(U)$ to $\Lambda^{k}(f)(x)$. Since the norm is semicontinuous in the Banach space $L_{q}$, the left-hand side of the inequality can be estimated as

$$
\int_{E_{\nu} \cap B\left(x_{0}, r\right)}\left|\Lambda^{k} f(x)\right|^{q} d x \leqslant \varliminf_{m \rightarrow \infty} \int_{E_{\nu} \cap B\left(x_{0}, r\right)}\left|\Lambda^{k} f_{m}(x)\right|^{q} d x .
$$

We have also $\int_{B\left(x_{0}, r\right)}\left|J\left(x, f_{m}\right)\right| d x \leqslant \mid f_{m}\left(B\left(x_{0}, r\right) \mid\right.$.

Since $\left|f\left(B\left(x_{0}, r\right)\right)\right|<\infty$ and the mapping $f$ is a homeomorphism, the images $f\left(S\left(x_{0}, r\right)\right)$ of the spheres $S\left(x_{0}, r\right)$ do not intersect under different 
$r$. It follows that the $n$-measure of the image of any sphere is zero for almost all $r:\left|f\left(S\left(x_{0}, r\right)\right)\right|=0$. We fix $r$ so that

$$
\left|f\left(S\left(x_{0}, r\right)\right)\right|=0
$$

and surround the image of the sphere $f\left(S\left(x_{0}, r\right)\right)$ by an $\varepsilon$-neighborhood $U_{\varepsilon}$. Since the mappings $f_{m}$ converge locally uniformly to the mapping $f$, it follows that, starting from a number $m_{0}$, the images of the spheres $f_{m}\left(S\left(x_{0}, r\right)\right), m \geqslant m_{0}$, are contained in this $\varepsilon$-neighborhood. It is clear that $\left|U_{\varepsilon}\right| \rightarrow 0$ as $\varepsilon \rightarrow 0$, and hence $\mid f_{m}\left(B\left(x_{0}, r\right)|\rightarrow| f\left(B\left(x_{0}, r\right) \mid\right.\right.$ as $m \rightarrow \infty$.

Taking into account that $M_{m}(x)^{\varkappa}$ converge in the biting sense to $M(x)^{\varkappa}$, we pass to the lower limit in (7) as $m \rightarrow \infty$. We get

$$
\int_{B\left(x_{0}, r\right)}\left|\Lambda^{k} f(x)\right|^{q} \chi_{E_{\nu}}(x) d x \leqslant\left(\int_{B\left(x_{0}, r\right)} M^{\varkappa}(x) \chi_{E_{\nu}}(x) d x\right)^{\frac{q}{\varkappa}} \mid f\left(\left.B\left(x_{0}, r\right)\right|^{\frac{q}{p}} .\right.
$$

Dividing both sides of this inequality by the measure of the ball $B\left(x_{0}, r\right)$, we obtain the following inequality

$$
\begin{aligned}
& \frac{1}{\left|B\left(x_{0}, r\right)\right|} \int_{B\left(x_{0}, r\right)}\left|\Lambda^{k} f(x)\right|^{q} \chi_{E_{\nu}}(x) d x \leqslant \\
& \quad \leqslant\left(\frac{1}{\left|B\left(x_{0}, r\right)\right|} \int_{B\left(x_{0}, r\right)} M^{\varkappa}(x) \chi_{E_{\nu}}(x) d x\right)^{\frac{q}{\varkappa}}\left(\frac{\mid f\left(B\left(x_{0}, r\right) \mid\right.}{\left|B\left(x_{0}, r\right)\right|}\right)^{\frac{q}{p}} .
\end{aligned}
$$

Since the homeomorphism $f$ is Sobolev differentiable, then by [13, Section 2.3 , formula $(2.5)]$ we have

$$
\frac{\mid f\left(B\left(x_{0}, r\right) \mid\right.}{\left|B\left(x_{0}, r\right)\right|} \rightarrow\left|J\left(x_{0}, f\right)\right| \text { as } r \rightarrow 0 \text { for almost all } x_{0} \in E_{\nu} .
$$

Hence, by the Lebesgue differentiability theorem, letting $r$ go to 0 we obtain that

$$
\left|\Lambda^{k} f(x)\right|^{q} \leqslant M^{q}(x)|J(x, f)|^{\frac{q}{p}} \text { for almost all } x \in E_{\nu} .
$$

As $U=\bigcup_{\nu} E_{\nu}$, the point-wise inequality (9) holds in $U$ almost everywhere.

In the case $1<q=p<\infty$ we can assume that a sequence of numbers $\left\{\left\|M_{m} \mid L_{\infty}(U)\right\|\right\}_{m \in \mathbb{N}}$ converges to $M=\varliminf_{m \rightarrow \infty}\left\|M_{m} \mid L_{\infty}(U)\right\| \in \mathbb{R}$. In 
this case, instead of (7), for any $\varepsilon>0$ there exists $m_{1}$ such that for all $m \geqslant m_{1}$ we have

$$
\int_{B\left(x_{0}, r\right)}\left|\Lambda^{k} f_{m}(x)\right|^{q} d x \leqslant(M+\varepsilon)\left(\int_{B\left(x_{0}, r\right)}\left|J\left(x, f_{m}\right)\right| d x\right) .
$$

Further, proceeding as in the case $q<p$, we obtain the estimation

$$
K_{k, p}(x, f) \leqslant(M+\varepsilon) \text { for almost all } x \in U .
$$

Since $\varepsilon>0$ is an arbitrary number, we get the desired estimation.

By the Corollary 1 , we have proved that the limit mapping $f$ belongs to $\mathcal{C D}_{q, p}^{k}(U ; W)$.

As a straightforward consequence of Theorem 4 we get the following

Corollary. [16] Let $f_{m} \in \mathcal{C D}_{q, p}^{k}(U ; W), m \in \mathbb{N}$, be a sequence of homeomorphisms of the Sobolev class $W_{l, \text { loc }}^{1}(U)$ with $k<l, q \leqslant l / k, 1<q \leqslant$ $\leqslant p<\infty$. Suppose that the sequence $f_{m}$ is locally bounded in $W_{l}^{1}(U)$, and converge locally uniformly to a homeomorphism $f: U \rightarrow W$ as $m \rightarrow \infty$. Assume also that there exists a function $M(x) \in L_{\varkappa}(U), \frac{1}{\varkappa}=\frac{1}{q}-\frac{1}{p}$, such that

$$
K_{k, p}\left(\cdot, f_{m}\right)(x) \leqslant M(x) \text { for all } m \in \mathbb{N}
$$

in $U$ almost everywhere. Then the limit mapping $f$ belongs to $\mathcal{C D}_{q, p}^{k}(U ; W)$ and $K_{k, p}(\cdot, f) \in L_{\varkappa}(U)$, where $\frac{1}{\varkappa}=\frac{1}{q}-\frac{1}{p}$.

Moreover, the inequality $K_{k, p}(x, f) \leqslant M(x)$ holds almost everywhere.

Remark. There exists a misprint in the paper [16]: in the statement of the main result the condition $q \leqslant l / k$ is missing.

Acknowledgment. This work was supported by Russian Foundation for Basic Research, agreement 17-01-00875 for the first author, and by the program of fundamental scientific researches of the SB RAS I.1.2., project 0314-2016-0006 for the second author.

\section{References}

[1] Baykin A. N., Vodop'yanov S. K., Capacity estimates, Liouville's theorem, and singularity removal for mappings with bounded ( $p, q)$-distortion. Sib. Math. J., 2015, vol. 56, no. 2, pp. 237-261.

DOI: http://doi.org/10.1134/S0037446615020056. 
[2] Brooks J. K., Chacon R. V., Continuity and compactness of measures. Adv. Math., 1980, vol. 37, no.1, pp. 16-26.

DOI: http://doi.org/10.1016/0001-8708(80)90023-7.

[3] Federer H. Geometric Measure Theory. Springer-Verlag, 1969.

[4] Fusco N., Moscariello G., Sbordone C., The limit of $W^{1,1}$ homeomorphisms with finite distortion. Calc. Var., 2008, vol. 33, no. 3, pp. 377-390.

DOI: http://doi.org/10.1007/s00526-008-0169-2.

[5] Gehring F. W., Iwaniec T., The limit of mappings with finite distortion. Ann. Acad. Sci. Fenn. Math., 1999, vol. 24, pp. 253-264.

[6] Hajłash P., Change of variables formula under minimal assumptions. Colloq. Math., 1993, vol. 64, no. 1, pp. 93-101.

DOI: http://doi.org/10.4064/cm-64-1-93-101.

[7] Iwaniec T., Šverák V., On mappings with integrable dilatation. Proc. Amer. Math. Soc., 1993, vol. 118, no. 1, pp. 181-188.

DOI: http://doi.org/10.1090/S0002-9939-1993-1160301-5.

[8] Reshetnyak Yu. G. Space Mappings with Bounded Distortion. Amer. Math. Soc., 1989.

[9] Stein E. M. Singular Integrals and Differentiability Properties of Functions. Princeton Univ. Press, 1970.

[10] Troyanov M., Vodop'yanov S. K., Liouville type theorems for mappings with bounded (co)-distortion. Ann. Inst. Fourier, Grenoble. 2001. vol. 52, no. 6, pp. 1753-1784.

[11] Vodop'yanov S. K., Closure of classes of mappings with bounded distortion on Carnot groups. Sib. Adv. Math., 2004, vol. 14, no. 1, pp. 84-125.

[12] Vodop'yanov S. K., Spaces of differential forms and maps with controlled distortion. Izvestiya: Mathematics, 2010, vol. 74, no. 4, pp. 663-689.

DOI: http://doi .org/10.1070/IM2010v074n04ABEH002502.

[13] Vodop'yanov S. K., Regularity of mappings inverse to Sobolev mappings. Sbornik: Mathematics, 2012, vol. 203, no. 10, pp. 1383-1410.

DOI: http://doi .org/10.1070/SM2012v203n10ABEH004269.

[14] Vodop'yanov S. K., Quasiconformal analysis of two-indexed scale of spatial mappings and its applications. Abstracts International Conference on Complex Analysis and its Applications (Krasnodar, June, 02-09), 2018, pp. 25-27.

[15] Vodop'yanov S. K., Gol'dstein V. M., Quasiconformal mappings and spaces of functions with generalized first derivatives. Sib. Math. J., 1976, vol. 17, no. 3, pp. 399-411. DOI: http://doi.org/10.1007/BF00967859. 
[16] Vodop'yanov S. K., Kudryavtseva N. A., On the convergence of mappings with $k$-finite distortion. Math. Notes, 2017, vol. 102, no. 5-6, pp. 878-883. DOI: http://doi.org/10.1134/S0001434617110281.

[17] Vodopyanov S. K., Molchanova A. O., Lower semicontinuity of mappings with bounded $(\theta, 1)$-weighted $(p, q)$-distortion. Sib. Math. J., 2016, vol. 57, no. 5, pp. 778-787. DOI: http://doi.org/10.1134/S0037446616050062.

Received May 31, 2018.

In revised form, June 18, 2018.

Accepted September 18, 2018.

Published online September 27, 2018.

N. A. Kudryavtseva

Novosibirsk State University

1 Pirogova str., Novosibirsk 630090, Russia

E-mail: nkudr@itam.nsc.ru

S. K. Vodopyanov

Sobolev Institute of Mathematics

4 Akademika Koptyuga pr., Novosibirsk 630090, Russia

E-mail: vodopis@math.nsc.ru 